\title{
Electrical and structural properties of p-type nanocrystalline silicon grown by LEPECVD for photovoltaic applications
}

\author{
Gabriel Micard ${ }^{*},{ }^{2}$ Giso Hahn ${ }^{1}$, Barbara Terheiden ${ }^{1}$, Daniel Chrastina ${ }^{2}$, Giovanni Isella ${ }^{2}$, \\ Tamara Moiseev ${ }^{3}$, Daniela Cavalcoli ${ }^{3}$, Anna Cavallini $i^{3}$, Simona Binetti ${ }^{4}$, Maurizio Acciarri ${ }^{4}$, \\ Alessia Le Donne, ${ }^{4}$ Michael Texier ${ }^{5}$, and Bernard Pichaud ${ }^{5}$ \\ ${ }^{1}$ Department of Physics, Faculty of Sciences, University of Konstanz, Jacob-Burckhardt-Str. 29, 78464 Konstanz, Germany \\ ${ }^{2}$ L-NESS, Dipartimento di Fisica del Politecnico di Milano, Polo Regionale di Como, Via Anzani 42, 22100 Como, Italy \\ ${ }^{3}$ Department of Physics, University of Bologna, viale C. Berti-Pichat 6/II, 40127 Bologna, ltaly \\ ${ }^{4}$ Dipartimento di Scienza dei Materiali, Università di Milano-Bicocca,Via Cozzi 53, 20125 Milano, Italy \\ ${ }^{5}$ Institut Matériaux, Microélectronique, Nanosciences de Provence (IM2NP UMR 6242) Fac. Sciences et Techniques de Saint Jérôme, \\ 262 Av. Escadrille Normandie-Niemen, 13397 Marseille, France
}

PACS 68.37.Og, 68.37.Ps, 68.55.ag, 73.63.Bd, 81.15.Gh, 84.60.Jt

* Corresponding author: e-mail gabriel.micard@uni-konstanz.de, Phone: +49 753188 2080, Fax: +49 7531883895

p-doped hy drogenated nanoctystalline slicon (p-ne-Sith) is one of the nost critical layers in thin film silicon solar cells. LEPECVD is a new technique for the growth of nc-Si at high growth rate without compromising the layer quality, using a dense but low energy plasma. Thin p-nc-siall layers are grown on glass and 2 nO 11 coated glass and heir structural and electrical properties are investigated as a function of the silane dilution (d) and of the doping ratio (DR). The influence of the substrate on the structural properties is investigated and discissed The incubation layer is clearly observed on both substrate types and its thickness is estimated. I WPECVI distinguishes itself from other high growtl rate methods by a very low impurity distribution coefficient to obtain a comparable conductivity and boron density. The conduction path is shown to be dependent on the density of bo: ron in the lajer while a significant decrease of conductiity at high DR is not observed in the studied range.
1 Introduction Hydrogenated nanocrystalline silicon (nc-Si:H) deposited at low temperature $\left(<300^{\circ} \mathrm{C}\right)$ is a promising material for device applications such as stable thin film solar cells or fast thin film transistors [1]. In this framework, low-energy plasma-enhanced chemical vapor deposition (LEPECVD) [2], like HWCVD (Hot Wire CVD) and VHF (Very High Frequency)-PECVD, is one of the new techniques developed for growing nc-Si:H at high enough deposition rates to address one of the most important obstacles towards its industrial application: the long deposition time required by standard rf-PECVD.

LEPECVD has already demonstrated growth rates of up to $5-10 \mathrm{~nm} / \mathrm{s}$ [2] depositing high-quality epitaxial $\mathrm{Si}$ films using a low-voltage, high-current dc arc discharge. Due to low ion energies, a high plasma density can be magnetically confined on the substrate surface without inducing defects in the deposited film.
In this paper we present the results of the structural characterization by means of AFM (Atomic Force Microscopy), c(conductive)-AFM, TEM (Transmission Electron Microscopy), HR(High Resolution)TEM and Raman spectroscopy as well as electrical characterization by temperature dependant lateral conductivity measurement and SIMS (Secondary Ion Mass Spectroscopy) on p-layers grown by LEPECVD.

A special emphasis is put on the incubation layer investigation identified by HRTEM and SIMS measurements

Transport mechanisms and dopant incorporation are also investigated establishing links to the microstructure.

\section{Experimental}

2.1 LEPECVD This method is based on an Ar plasma created by a Ta filament in a plasma ingnition chamber and then transmitted into the growth chamber through an ori- 
fice. The plasma focusing towards the sample is obtained by means of an anode ring. A negative dc potential is applied to the filament while the chamber and anode are grounded such that a large direct current flows through the plasma (typically $50 \mathrm{~A}$ ). The plasma is confined onto the substrate (suspended beneath a heater) by a magnetic field created by a set of coils wrapped around the chamber.

The precursors gases, $\mathrm{SiH}_{4}$ and $\mathrm{B}_{2} \mathrm{H}_{6}$ (diluted in Ar, for doping), along with $\mathrm{H}_{2}$ can be introduced into the chamber and cracked into reactive radicals by the plasma [3] but since the arc voltage is only $20-30 \mathrm{~V}$ the ion energy is low enough that the growing film is not damaged. The creation of radicals by the plasma means that the growth process is quite efficient, with about $5-10 \%$ of the growth precursors being incorporated into the growing film depending on the size of the substrate, regardless of the substrate temperature [3]. The base pressure of the system is $10^{-9} \mathrm{mbar}$, while the working pressure is roughly $10^{-2}$ mbar.

2.2 Sample preparation In the case of LEPECVD, it is important to note that several parameters such as arc discharge current, confinement coil current and substrate temperature, influence mainly the spatial uniformity and film sticking properties. Therefore, these parameters are set to optimal values that are the same for all depositions. The silane flow influences the growth rate in a quasi proportional relationship, therefore we maintain it at $0.5 \mathrm{sccm}$ for all experiments.

In the first sample series we grew p-layers on bare and $\mathrm{ZnO}: \mathrm{Al}$ coated 7059 Corning glass substrate $\left(5 \times 5 \mathrm{~cm}^{2}\right)$ at a constant doping ratio $\left(\mathrm{DR}=\left[\mathrm{B}_{2} \mathrm{H}_{6}\right] /\left[\mathrm{SiH}_{4}\right]\right)$ of $9 \%$ and growth time of $12 \mathrm{~min}$, varying the silane dilution $\left(d=\left[\mathrm{SiH}_{4}\right] /\left(\left[\mathrm{SiH}_{4}\right]+\left[\mathrm{H}_{2}\right]\right)\right)$ from 1 to $10 \%$. In a second sample series, entirely grown on bare glass substrate, we kept the silane dilution $d$ constant at $1 \%$, reduced the growth time to $6 \mathrm{~min}$ in order to study thinner layers, and varied the DR between 0.4 and $9 \%$.

2.3 Measurements The fully amorphous layer at the

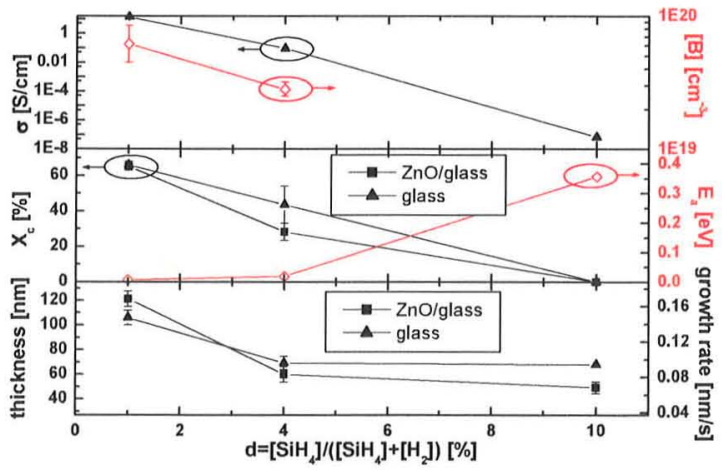

Figure 1 Dilution series on $\mathrm{ZnO} /$ glass and bare glass substrates. Error bars on $\chi_{c}$ and thickness refer to spatial distribution over the substrate while on [B], they refer to an average value over the thickness excluding the incubation layer. beginning of the growth, the so-called incubation layer [4], is seen in TEM and HRTEM pictures as well as SIMS measurements $\left(\mathrm{O}_{2}{ }^{+}\right.$ion beam $)$. The crystalline fraction $\chi_{\mathrm{c}}$ is investigated by Raman spectroscopy while morphology related to the conduction mechanism and paths is investigated by a combination of AFM, c-AFM, and a macroscopic dark conductivity $\sigma$ study dependent on temperature. AFM and C-AFM analyses were carried out in a Solver

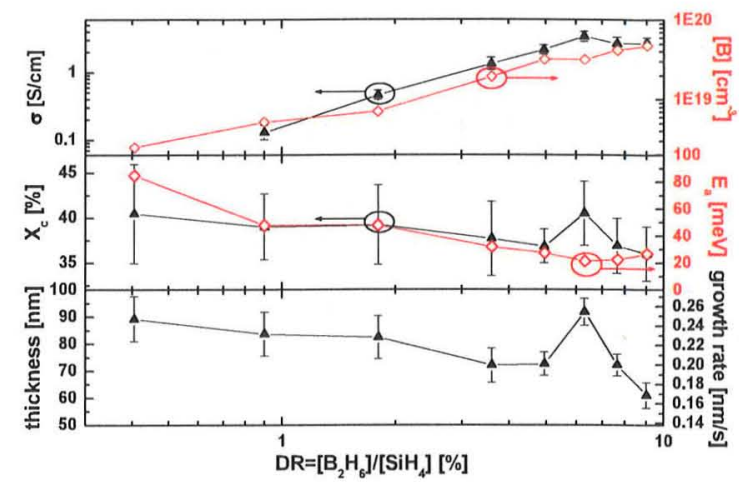

Figure 2 Doping ratio series on bare glass substrates. Error bars on $\chi_{c}$, thickness and conductivity refer to spatial distribution over the substrate while on [B], they refer to an average value over the

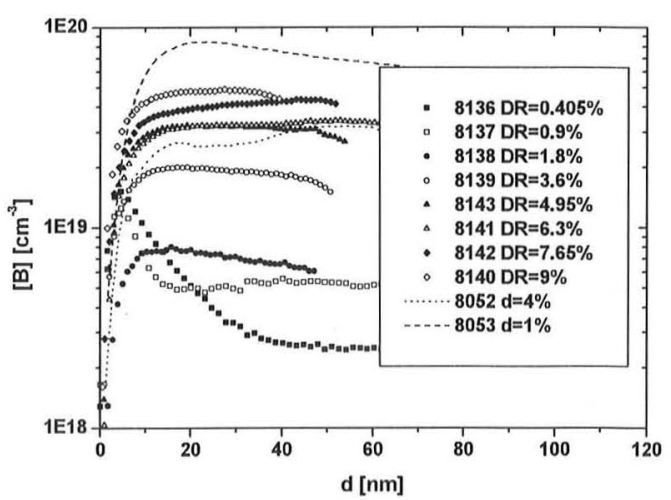

Figure 3 SIMS boron concentration depth profiles of samples of the $d$ and DR series starting from the glass/nc-Si interface.

P47H-Pro from NT-MDT in constant force mode (contact mode) in air at small load conditions (normal force lower than $600 \mathrm{nN}$ ). The constant positive bias applied to the probe was below $3 \mathrm{~V}$ in order to avoid a local anodic oxidation effect. Special care was used during the measurements in order to avoid significant artifact effects $[5,6]$.

An indicator for the reliability of the total boron concentration [B] extracted by SIMS is the constant value of the silicon signal. According to this criterion, the values extracted at the beginning of the measurement (sample surface) are not reliable down to around 
$20 \mathrm{~nm}$ inside the nc-Si. A gold layer was deposited on top of some samples to improve this aspect. The SIMS data are, however, reliable around the substrate/silicon interface until the signal drops down at the substrate. The profiles in Fig. 3 are reversed so that they start from the substrate/silicon interface. We set the interface position at the point where the value of the silicon SIMS signal drops by two orders of magnitude with respect to the plateau signal. Our lowest conductivity sample showed a continuously varying silicon signal, probably due to charging effects, and is thus not reliably measured and not displayed here.

The same measurement methods applied for the first series of samples were performed for the second series except for TEM, while four point probe measurements of the conductivity at room temperature were performed in addition on 9 points per sample to map the spatial distribution.

The extraction of the activation energy of the dark conductivity $E_{a}$ is surely underestimated due to a lack of conductivity data above $340 \mathrm{~K}$ and a non negligible variable range hopping, which is far less temperature activated, up to relatively high temperatures. The extracted values give, however, a reliable trend for both series.

The thickness of all samples was assessed by fitting the reflectivity data using OPTICAL software [7] for 5 or 9 points per sample.

\section{Results}

3.1 Dilution series In general, decreasing the dilution from 10 to $1 \%$ leads to a large increase in $\chi_{c}$, in $\sigma$, in [B] and an expected decrease in $E_{a}$ (Fig. 1). The sample at $d=1 \%$ showed $\chi_{c}=70 \%, \sigma=14 \mathrm{~S} / \mathrm{cm}$ with $E_{a}=8 \mathrm{meV}$ and $[\mathrm{B}]=6 \times 10^{19} \mathrm{~cm}^{-3}$. From the low value of $E_{a}$ close to zero in this sample we conclude that the conduction occurs only in the degenerated crystalline phase.

TEM observations showed the presence of an incubation layer [4], which is $10 \mathrm{~nm}$ and $5 \mathrm{~nm}$ thick on glass and $\mathrm{ZnO}$ /glass substrate, respectively (Fig. 4). Electrons of the plasma are likely to flow easily through the $\mathrm{ZnO}$ coating, thanks to its high conductivity, thus inducing an additional heating source that may explain the appearance of crystallites in the Si layer earlier in the growth process. The incubation layer is followed by an intermediate region composed of scattered misaligned nanocrystals in an amorphous matrix that is $30 \mathrm{~nm}$ thick for $\mathrm{ZnO} /$ glass substrate and almost non-existent for samples on glass. This effect could be perhaps explained by the higher roughness of the

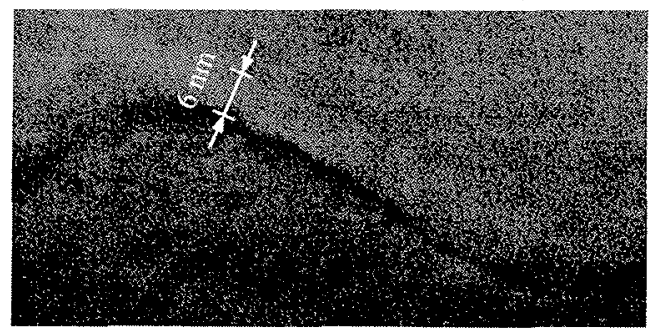

Figure 4 Cross section TEM image of the incubation layer.
$\mathrm{ZnO} /$ glass substrate that favours misaligned growth and slows down a global organisation of the crystalline phase. Then begins a columnar or conical growth with columns of diameter 2 to $15 \mathrm{~nm}$ (Fig. 5). Therefore, the relatively lower global crystallinity of $\mathrm{ZnO} /$ glass samples in comparison to glass substrate samples could be explained by this delay of dense crystallisation induced by the rougher $\mathrm{ZnO}$ surface.

3.2 DR series In general, increasing the DR leads to a slight decrease in global crystallinity and in growth rate (Fig. 2), probably due to the difficulty in incorporating boron in the layer and its amorphisation properties.

A clear proportionality has been established between the relative density of boron inside the film and the DR, corresponding to the impurity distribution coefficient $d_{l}$ defined as $\left[C_{B}\right]_{\text {film }} /\left[C_{B}\right]_{\text {gas }}$ where $\left[C_{B}\right]_{\text {film }}$ is the boron atomic concentration in the film and $\left[\mathrm{C}_{\mathrm{B}}\right]_{\text {gas }}$ is the molecular concentration of the dopant in the plasma [8]. The $d_{l}$ value is, however, surprisingly low $(\sim 0.01)$, which is currently unexplained, in comparison to the observed value for nc-Si grown by rf-PECVD ( 0.1) [9]. This value, however, reaches almost 1 for a-Si [9] corresponding to a higher incorporation but a lower dopant activation which in the end is causing a lower conductivity of a-Si compared to nc-Si.

While the AFM map (Fig. 6) shows a cluster segmentation of the surface, the relatively large hillock size probably means that they are composed of several nanocrystals. Superposing the C-AFM maps reveals that, unlike the case of LEPECVD grown intrinsic nc-Si layers [10], the microscopic conduction occurs mainly at the hillock boundaries (Fig. 6). Increasing the DR, we observe that the conduction occurs also on several hillocks (Fig. 7).

A tentative explanation could be that the cluster boundaries act as preferential incorporation sites for impurities, which in the case of intrinsic nc-Si are detrimental to the conduction process. This is in contrast to doped nc-Si, providing boron present at the cluster boundaries is activated. It is thus likely that there should be a significant difference in nanocrystal size and/or spacing between them, depending if they are located in the clusters or in the boundaries.

The phenomenon of layer amorphisation by boron while increasing the DR could be explained in terms of reducing the grain size that increases the fraction of amorphous tissue [11]. Increasing the DR is likely to reduce and homogenize the grain size that finally allows also significant conduction inside the clusters. For low DR samples $(\mathrm{DR}=0.4$ or $0.9 \%)$ we observe a clear maximum in the boron density at the beginning of the growth for $\approx 10 \mathrm{~nm}$ (Fig. 3). Considering that the impurity distribution coefficient is one decade higher for a-Si than for $\mathrm{nc}-\mathrm{Si}$, this region is probably amorphous, corresponding to the incubation layer, and its thickness is in agreement with the estimation obtained in Section 3.2 by HRTEM. Increasing the $\mathrm{DR}$, however, this phenomenon is likely to be covered by a 

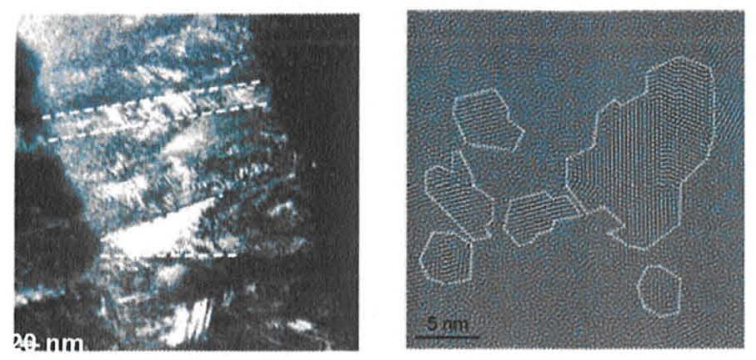

Figure 5 Cross-section dark-field TEM micrograph showing the columnar structure (left) and filtered HRTEM image of nanocrystals observed near the substrate/Si interface (right)

higher boron density at cluster boundaries and finally in the clusters themselves.

It is interesting to observe that there is a slight evolution of the plateau concentration during the growth process (Fig. 3): slightly decreasing [B] for samples with DR 1.8 to $6.3 \%$ and slightly increasing [B] for higher DR. This leads to think that, taken apart the incubation layer, below $\mathrm{DR}=6.3 \%$ the crystallinity slightly increases with thickness, starting from relatively low values, while above $\mathrm{DR}=6.3 \%$ it slightly decreases with thickness while starting at relatively higher values. This is in agreement with the trend observed in activation energy that shows a slight minimum value at $\mathrm{DR} \approx 6.3 \%$ correlated with the highest crystallinity value of the set. It is, however, not clear why the crystallinity value obtained for $\mathrm{DR}=6.3 \%$ is so high in comparison to the other samples.

4 Discussion The comparison of the sample $d=1 \%$ in the dilution series with the sample DR $=9 \%$ in the doping ratio series shows a very large discrepancy in growth rate despite their intended identical growth parameters. While interpretation of results is consistent within each series, the differing results for the two samples originating from different series, but grown using the same parameters, cannot be compared for a reason which is still unclear. While outstanding and consistent results were obtained for the sample of $d=1 \%$ in the dilution series, 'only' standard state of the art values were obtained for the sample $\mathrm{DR}=9 \%$ of the DR series.

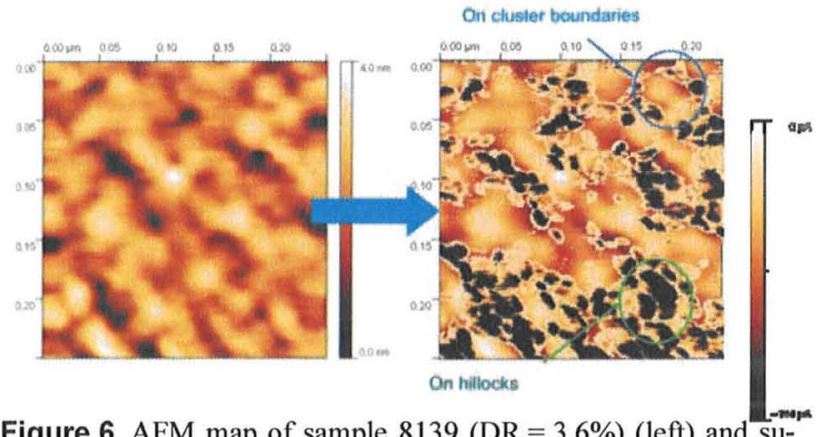

Figure 6 AFM map of sample 8139 (DR $=3.6 \%$ ) (left) and superposition with its conductive AFM map (right).

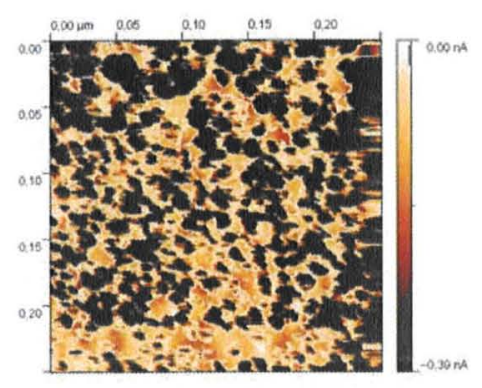

Figure 7 Superposition of AFM and c-AFM map of sample 8140 $(\mathrm{DR}=9 \%)$.

5 Conclusion This study demonstrated clearly the presence of the incubation layer by both TEM and SIMS, and its thickness on $\mathrm{ZnO} /$ glass and glass substrates could be estimated. We observed also an increase of homogeneity in the in-plane boron density when increasing the DR. From the DR series, the highest conductivity is obtained in the case of the most homogeneous in depth boron distribution and we suppose that this might correspond to a threshold between a cluster boundary conduction type and a mixed conduction type involving the clusters themselves as well. The study highlighted also a very low impurity distribution coefficient in comparison with other growth techniques that translates into a maximum of the conductivity obtained for a very high value of DR $(6.3 \%)$.

Acknowledgements Part of this work was supported by the EU in the frame of the NANOPHOTO project (NMP3-CT2005-013944) which is gratefully acknowledged.

\section{References}

[1] R. Rizzoli, C. Summonte, J. Pl'a, E. Centurioni, G. Ruani, A. Desalvo, and F. Zignani, Thin Solid Films 383, 7-10 (2001).

[2] C. Rosenblad, H. von Känel, M. Kummer, A. Dommann, and E. Müller, Appl. Phys. Lett. 76, 427 (2000).

[3] M. Rondanini, S. Cereda, F. Montalenti, L. Miglio, and C. Cavallotti, Surf. Coat. Technol. 201, 8863 (2007).

[4] T. Fujibayashi and M. Kondo, J. Appl. Phys. 99, 043703 (2006).

[5] D. Cavalcoli, M. Rossi, A. Tomasi, and A. Cavallini, Nanotechnology 20, 045702-045708 (2009).

[6] B. Rezek, J. Stuchlýk, A. Fejfar, and J. Kocka, Appl. Phys. Lett. 92, 587 (2002).

[7] E. Centurioni, Appl. Optics 44, 7532 (2005).

[8] R. A. Street, Hydrogenated Amorphous Silicon (Cambridge University Press, Cambridge, 1991), p. 139

[9] P. Alpuim, V. Chu, and J. P. Conde, J. Appl. Phys. 86, 3812 (1999).

[10] A. Cavallini and D. Cavalcoli, Scanning 30, 1 (2008).

[11] L. E. Ramos, E. Degoli, G. Cantele, S. Ossicini, D. Ninno, J. Furthmüller. and F. Bechstedt, J. Phys.: Condens. Matter 19, 466211 (2007). 